P0-0816 TEMPERAMENT IN VERY PRETERM INFANTS AT 2YEARS CORRECTED AGE: VALIDATION OF THE INFANT CHARACTERISTIC QUESTIONNAIRE IN THE ITALIAN ACTION COHORT

${ }^{1}$ C Croci, ${ }^{2} \mathrm{P}$ Ferrante, ${ }^{3} \mathrm{~V}$ Carnielli, ${ }^{4} \mathrm{~V}$ Chiandotto, ${ }^{5} \mathrm{D}$ Di Lallo, ${ }^{6} \mathrm{MC}$ Fertz, ${ }^{7} \mathrm{~S}$ Miniaci, ${ }^{8} \mathrm{~F}$ Rusconi, ${ }^{1} \mathrm{M}$ Cuttini. ${ }^{1}$ Research Unit of Perinatal Epidemiology, Pediatric Hospital Bambino Gesu', Rome, Italy; ${ }^{2}$ Occupational Medicine Department (DML), National Workers Compensation Authority (INAIL), Rome, Italy; ${ }^{3}$ Maternal and Child Health Institute, Marche University and Salesi Hospital, Ancona, Italy; ${ }^{4}$ Neonatal Intensive Care Unit, S. Maria Della Misericordia University Hospital, Udine, Italy; ${ }^{5}$ Hospital Network Planning and Research Area, Lazio Region, Rome, Italy; ${ }^{6}$ Neonatal Intensive Care Unit, Burlo Garofalo Maternal and Child Health Institute, Trieste, Italy; ${ }^{7}$ Neonatal Intensive Care Unit, Pugliese-Ciaccio Hospital, Catanzaro, Italy; ${ }^{8}$ Unit of Epidemiology, Anna Meyer Children's University Hospital and Regional Agency for Health of Tuscany, Florence, Italy

10.1136/archdischild-2014-307384.1450

Background and aims Temperament in very preterm infants (VPI) has been rarely studied. We used a short version of the Bates Infant Characteristic Questionnaire (ICQ) to explore maternal reported child difficultness in an area-based cohort of Italian VPI.

Methods Study population included 1196 infants (response rate $85 \%)$. A medical examination was carried out to assess infants' health and presence of disabilities. A postal maternal questionnaire was used to measure development and temperament. We used the sections corresponding to 'Fussy-difficult' and 'Unadaptable', with 12 items rated on a 7-point Likert-type scale. Higher scores indicate more problematic temperament. Statistical analysis included factor analysis and calculation of Cronbach alpha. To assess validity, comparisons with sleep and eating problems reported by mothers independently from ICQ were carried out.

Results For this study only singletons born from Italian mothers were considered (n. 586); 55\% were males, and 21\% were below 28 weeks gestation. Forty infants $(7 \%)$ had at least one severe disability. Mean ICQ score was 34.1 (SD 10.1), and median 33 (range 13-69). Factor loadings were comparable to the original English version, and internal consistency was satisfactory (alpha 0.88 for fussy-difficult and 0.71 for Unadaptable). Infants with difficulties getting to sleep, frequent night awakenings, and 'picky eaters' had significantly higher ICQ scores ( $p$ values $<0.01$ ).

Conclusion The short version of the ICQ may represent a quick and easily administered tool for the identification of difficult temperament traits in clinical and research settings.

Acknowledgements Action follow-up study was funded by Italian $\mathrm{MoH}$ and Chiesi Spa.

\section{PO-0817 MEASURING BEHAVIOURAL PROBLEMS IN VERY PRETERM CHILDREN: COMPARISON OF SDQ AND CBCL RESULTS IN THE ITALIAN ACTION COHORT}

G Riccio, M Lacchei, C Giorno, C Rosa, G Giana, I Croci, M Cuttini. Research Unit of Perinatal Epidemiology, Pediatric Hospital Bambino Gesu', Rome, Italy

10.1136/archdischild-2014-307384.1451

Background and aims Very preterm children have increased risk of behavioural problems. Easily administered valid screening tests are required for epidemiological studies. This study aims at exploring the relation between the Child Behaviour Checklist (CBCL) and the shorter Strengths and Difficulties Questionnaire (SDQ) in a subsample of the Italian area-based ACTION cohort followed up to school age.
Methods The ACTION follow-up study uses the SDQ parent version to assess the emotional and behavioural problems of children born $<32$ weeks gestation and survived to school age. For the purposes of the study, a subsample (n.223) of the cohort recruited in Lazio region was administered both SDQ and CBCL, parent-reported. We computed Spearman's rho coefficients to carry out correlation analyses. The K-Cohen Test was used to measure agreement SDQ and CBCL instruments

Results 121 children were males (54.3\%). 44 (19.7\%) were $<28$ weeks gestation. Seven had cerebral palsy, 4 were blind or almost blind, and 9 used hearing aids.

According to CBCL, 20 children (9\%) had significant and 18 $(8.1 \%)$ borderline difficulties. SDQ identified as pathological 14 children (6.3\%), while 13 were classified as borderline $(5.8 \%)$. Overall agreement was $86.1 \%$ (Kappa 0.47, p < 0.0001).

Spearman's rho was 0.63 for emotional/internalising problems, 0.61 for behavioural/externalising, and 0.74 for total problems. All values were statistically significant $(\mathrm{p}<0.001)$.

Conclusions Correlation and inter-rater agreement between the two instruments are good. Overall, however, SDQ appeared more conservative in the identification of children with problems.

Acknowledgements The ACTION follow-up study was funded by Italian $\mathrm{MoH}$ and Chiesi Spa.

\section{PO-0818 A CASE SERIES STUDY ON CLINICAL PROFILE OF CHILDREN WITH NEUROCUTANEOUS SYNDROMES}

N Divakar. Paediatrics, Pushpagiri Institute of Medical Sciences, Cochin, India

\subsection{6/archdischild-2014-307384.1452}

Introduction Neurocutaneous syndromes (NCS) are a heterogenous group of disorders characterised by abnormalities of both the integument and central nervous system that are believed to originate from a defect in differentiation of the primitive ectoderm. Cutaneous manifestations usually appear early in life and progress with time, but neurological features generally present at a later age.

Aim To study the clinical profile of children with neurocutaneous syndromes and their various symptomatology, the seizure types and the response to treatment.

Subjects and methods A retrospective crosssectional study was conducted in the Department of Paediatrics, Pushpagiri Medical College Hospital, Tiruvalla, during the period from January 2013 to June 2013. Children between the age group 0 and 15 years were included in the study on the basis of standard diagnostic criteria for different NCS. Investigations done were CT, MRI, EEG, and skin biopsy for appropriate cases

Results The study population comprised of 10 children (5 boys, 5 girls). The various forms of NCS observed were Sturge Weber syndrome (SWS) - 4 Neurofibromatosis (NF1)- 2, Hypomelanosis of Ito (HOI) -2 , Tuberous sclerosis complex (TSC) -1 , and Incontinentia pigmenti (IP) -1 . A total of 8 children $(80 \%)$ presented with neurological symptoms and the remaining 2 (20\%) presented with cutaneous symptoms of which 1 was found to have learning disability on evaluation. The neurological problems were, $70 \%$ had seizures of which $100 \%$ were SWS and TSC, 50\% were HOI and NF1. 72\% had generalised tonic clonic seizures (GTCS) and 28\% had focal seizures. The child with TSC showed refractory epilepsy. Developmental delay was detected in 50\% of cases and maximum delay was seen in HOI. Family history of 
the same disease was obtained in 2 cases $(50 \%$ of NF1 and $100 \%$ of IP).

Conclusions The leading NCS in this study was SWS.70\% of children with NCS presented with seizures. The commonest type of seizure among them is GTCS. Children with TSC had seizures refractory to anticonvulsants. Careful evaluation of NCS children can reveal problems like Learning disabilities.

Recommendation All children with neurocutaneous markers should be evaluated and investigated in detail to detect neurological affection.

Keywords Neurocutaneous syndromes, Hypomelanosis of Ito, Sturge Weber Syndrome, Tuberous Sclerosis Complex.

\section{P0-0819 CASE SERIES OF CHILDREN WITH NEUROCUTANEOUS SYNDROMES}

N Divakar. Paediatrics, Pushpagiri Institute of Medical Sciences, Cochin, India

10.1136/archdischild-2014-307384.1453

Introduction Neurocutaneous syndromes (NCS) are a heterogenous group of disorders characterised by abnormalities of both the integument and central nervous system that are believed to originate from a defect in differentiation of the primitive ectoderm. Cutaneous manifestations usually appear early in life and progress with time, but neurological features generally present at a later age.

Aim To study the clinical profile of children with neurocutaneous syndromes and their various symptomatology, the seizure types and the response to treatment.

Subjects and methods A retrospective crosssectional study was conducted in the Department of Paediatrics, Pushpagiri Medical College Hospital, Tiruvalla, during the period from January 2013 to June 2013. Children between the age group 0 and 15 years were included in the study on the basis of standard diagnostic criteria for different NCS. Investigations done were CT, MRI, EEG,and skin biopsy for appropriate cases

Results The study population comprised of 10 children (5 boys, 5 girls). The various forms of NCS observed were Sturge Weber syndrome (SWS) - 4 Neurofibromatosis (NF1)- 2, Hypomelanosis of Ito (HOI) -2 , Tuberous sclerosis complex (TSC) -1 , and Incontinentia pigmenti (IP) -1 . A total of 8 children (80\%) presented with neurological symptoms and the remaining $2(20 \%)$ presented with cutaneous symptoms of which 1 was found to have learning disability on evaluation. The neurological problems were, $70 \%$ had seizures of which $100 \%$ were SWS and TSC, 50\% were HOI and NF1. 72\% had generalised tonic clonic seizures (GTCS) and 28\% had focal seizures. The child with TSC showed refractory epilepsy. Developmental delay was detected in 50\% of cases and maximum delay was seen in HOI. Family history of the same disease was obtained in 2 cases $(50 \%$ of NF1 and $100 \%$ of IP).

Conclusions The leading NCS in this study was SWS.70\% of children with NCS presented with seizures. The commonest type of seizure among them is GTCS. Children with TSC had seizures refractory to anticonvulsants. Careful evaluation of NCS children can reveal problems like Learning disabilities.

Recommendation All children with neurocutaneous markers should be evaluated and investigated in detail to detect neurological affection.

Keywords Neurocutaneous syndromes, Hypomelanosis of Ito, Sturge Weber Syndrome, Tuberous Sclerosis Complex.
P0-0820 SLEEP COMPLAINTS AMONG THE NEUROLOGICALLY IMPAIRED CHILDREN: QUESTIONNAIRE-BASED STUDY

${ }^{1} \mathrm{~T}$ Ediberidze, ${ }^{1} \mathrm{~N}$ Gogatishvili, ${ }^{2} \mathrm{~N}$ Tatishvili, ${ }^{3} \mathrm{~L}$ Maisuradze, ${ }^{1} \mathrm{~S}$ Kasradze. ${ }^{1}$ Epilepsy Centre, Institute of Neurology and Neuropsychology, Tbilisi, Georgia; '2Department of Neuroscience, M. Iashvili Children Central Hospital, Tbilisi, Georgia; ${ }^{3}$ llia State University, Tbilisi, Georgia

10.1136/archdischild-2014-307384.1454

Background and aims Many studies have shown a significant increase in SD in neurologically impaired patients. The aim of study was to evaluate the prevalence of sleep disturbances (SD) among the children with cerebral palsy (CP) with or without comorbidity with Epilepsy.

Methods A structured sleep-waking questionnaire was developed on the basis of Child Sleep Questionnaire for Parents and the Paediatric Sleep Questionnaire. Subjects were recruited from the Institute of Neurology and Neuropsychology/Tbilisi/Georgia.

Results 165 1-to-8-years-old children with CP and 359 age and sex-matched subjects were selected. 85 patients with CP had Epilepsy. Different SD were identified in $64.8 \%(\mathrm{n}=107)$ of children with CP vs. $20.3 \%(n=73)$ in healthy children. Sleep disorder breathing (SDB) was the commonest among the children with CP $58.78 \%(\mathrm{n}=97)$ vs. $8.07 \%(\mathrm{n}=29)$ in controls. Other main problems in neurologically impaired children were problems with sleep onset 41.2\% $(n=68)$ and sleep maintenance $39.4 \%(n=65)$. The frequency of both was about 4 -fold higher than in healthy controls. Sleep onset difficulty was commonest among controls $11.4 \%(n=41)$.

SDB was more prevalent among the children with $\mathrm{CP}$ and Epilepsy $69.4 \%(\mathrm{n}=59)$ vs. $47.5 \%(\mathrm{n}=38)$ in children without seizures. Problems with sleep onset and maintenance were a little higher among the subjects without Epilepsy 48.75\% ( $\mathrm{n}=$ $39)$ and $42.5 \%(n=34)$ correspondingly vs. $34.12 \%(n=29)$ and $36.47 \%(\mathrm{n}=31)$ in children with CP and Epilepsy.

Conclusions Study shows that SD is more prevalent in neurologically impaired children. Children with CP and Epilepsy show to be at higher risk to have SDB, compared to children with $\mathrm{CP}$ alone;

\section{P0-0821 THE APPROACH TO THE CHILD WITH EPILEPSY (APCWEP) AUDIT IN A BUSY UAE GENERAL HOSPITAL}

T El-Azzabi, V Malpani, MS Kasim, R Ravindran. Child Health Institute, Alain Hospital, AlAin, United Arab Emirates

\subsection{6/archdischild-2014-307384.1455}

Background and aims Epilepsy is a common neurological disorder. We are developing services for children with epilepsy (CWE) at Al-Ain hospital. Aiming to improve services we conducted an audit to evaluate the assessment of all CWE against NICE guidelines.

Methods Cases were identified from the hospital database. Retrospectively, electronic case notes of children presented with a diagnosis of seizure (s) between 1/7/2011 and 31/3/2014 were reviewed. Each note of all children meeting inclusion criteria was evaluated, twice independently, using modified Epilepsy 12 performance indicators.

Results 395 children were identified with a diagnosis of seizure(s). 91 fulfilled entry criteria.

1. 70/91 were referred to epilepsy-expert paediatricians.

2. 58/70 were seen within 2 weeks.

3. None seen by epilepsy nurse (Unavailable). 